\title{
Fármacos e alimentos: interações e influências na terapêutica
}

Drug-food interactions and their influence in pharmacotherapy

Márcia LOMBARDO, Jaqueline Kalleian ESERIAN

Instituto Adolfo Lutz, Centro de Medicamentos, Cosméticos e Saneantes. Avenida Doutor Arnaldo, 355, Prédio BQ, $5^{\circ}$ andar. CEP 01246-000 - São Paulo SP, Brasil. Email:mlombardo@ial.sp.gov.br

\section{ABSTRACT}

The occurrence of interactions between drugs and foods can be decisive events for achieving therapeutic success due to interferences in drug performance and also in nutrient uptake. The objective of this review was to highlight drugfood interactions with impact on health, and discuss about the appropriate measures to avoid or minimize them. Several authors point that the most important food-drug interactions are those that affect drug bioavailability. Fortunatelly, many of the interaction mechanisms have been elucidated. Therefore, food-drug interaction studies are important contributions to ensure the correct use of drugs and enable the development of suitable clinical protocols.

Key Words: food-drug interactions, diet, supplement food, pharmaceutical care

\section{RESUMO}

Interações entre fármacos e alimentos podem ser determinantes na obtenção do sucesso terapêutico, devido a interferências tanto no metabolismo de fármacos quanto na absorção de nutrientes. O objetivo deste trabalho foi realizar uma revisão bibliográfica sobre interações fármaco-alimento de impacto à saúde, bem como discutir as medidas adequadas para evitá-las ou minimizá-las. A literatura indica que as interações fármaco-alimento mais importantes são aquelas que afetam a biodisponibilidade do fármaco, sendo que muitas delas possuem mecanismos completamente elucidados. Estes estudos são importantes contribuições para o uso correto de medicamentos e possibilitam a elaboração de protocolos clínicos adequados.

Palavras Chave: interações alimento-fármaco, dieta, suplementação alimentar, atenção farmacêutica

\section{INTRODUÇÃO}

Inúmeras são as possibilidades de ocorrência de interações entre fármacos e alimentos, eventos estes frequentemente desconhecidos ou ignorados por profissionais e pacientes. De acordo com a literatura, as interações fármaco-alimento podem apresentar um caráter apenas teórico, mas também podem ocorrer interações que resultam em efeitos relevantes à prática clínica (1).

A administração de medicamentos junto das refeições pode ser requerida, seja para reduzir irritação da mucosa gastrointestinal, favorecer o cumprimento do horário da terapia, levar a um aumento desejado da absorção do fármaco ou mesmo manter concentrações plasmáticas efetivas do fármaco $(2,3)$. Por outro lado, algumas vezes tal rotina pode ser desvantajosa, devido a interferências na resposta farmacológica ou na manutenção do estado nutricional do paciente $(4,5)$.

Como impacto negativo na terapia medicamentosa, a diminuição da biodisponibilidade de fármacos pode resultar em falhas do tratamento, enquanto que o aumento da biodisponibilidade pode acarretar em sérios efeitos tóxicos (6).

Segundo Bushra et al (2011), as interações fármaco-alimento que geram reações adversas desnecessárias têm como principais consequências o aumento da morbidade e do tempo de hospitalização (4). Como a maioria dos medicamentos é administrada por via oral, interações entre fármacos e alimentos podem ser consideradas eventos facilitados. 
A simples presença de alimentos no estômago pode atrasar o esvaziamento gástrico e influenciar na absorção de fármacos $(2,3)$.

A secreção ácida ou biliar e a motilidade gastrointestinal são também variáveis que influenciam o potencial de interação, pois estão relacionadas com a solubilidade e biodisponibilidade dos fármacos e nutrientes (2).

Diversos fatores justificam a ocorrência de interações fármaco-alimento, como as características físico-químicas das substâncias envolvidas, a dose do fármaco e a quantidade de nutrientes disponíveis, o momento de administração do medicamento e o horário da dieta, além de aspectos individuais, como quadro clínico, polifarmácia, constituição enzimática e microflora intestinal (6). Interações fármaco-alimento são críticas no caso de pacientes suscetíveis e hospitalizados, principalmente no uso de fármacos de baixo índice terapêutico ou que necessitem ajuste de dose, pois pequenas mudanças na farmacocinética podem causar grandes interferências na eficácia e segurança do tratamento $(6,7)$.

Dessa forma, o presente trabalho teve por objetivo realizar uma revisão sobre interações fármaco-alimento significativas, destacando medidas preventivas e sua importância no cuidado à saúde.

\section{MÉTODO}

As buscas bibliográficas foram realizadas nas bases de dados Scielo e Medline, utilizando as seguintes palavras-chave: interação medicamento-alimento; interação alimento-droga; interação droga-nutriente; food-drug interaction; supplement-drug interaction. Como critérios de inclusão foram considerados artigos originais e revisões abordando o tema, no período de 1995 a 2013. Foram excluídas as publicações sobre interações de fármacos com plantas medicinais ou com nutrientes cuja origem alimentar não foi mencionada.

\section{RESULTADOS}

As interações fármaco-alimento mais frequentes são aquelas que afetam a taxa de absorção de fármacos, principalmente devido a proteínas, gorduras e fibras da dieta (4). Os aminoácidos provenientes de uma dieta rica em proteínas podem inibir a absorção de fármacos, como ocorre com os fármacos antiparkinsonianos levodopa e metildopa, enquanto o consumo excessivo de fibras pode reduzir a absorção de fármacos, como é o caso do cardiotônico digoxina. Refeições hiperlipídicas podem aumentar a absorção de fármacos e trazer riscos, a exemplo da carbamazepina (anticonvulsivante) ou mesmo ser requerida para favorecer a absorção de fármacos, como a griseofulvina (antifúngico) $(2,3)$.

Algumas interações fármaco-alimento podem ocorrer por reações químicas de complexação ou precipitação, as quais frequentemente são responsáveis pela diminuição da biodisponibilidade de fármacos e falhas no tratamento. $\mathrm{O} \mathrm{pH}$ da dieta também pode contribuir para redução da eficácia farmacológica, pois dependendo do caráter ácido ou básico da molécula, acelera a excreção de certos fármacos (Quadro 1).

Certas interações fármaco-alimento possuem mecanismos de ação específicos e bem definidos em estudos científicos (Quadro 2), como inibição enzimática, inativação, antagonismo e somação de efeitos, muitas vezes determinados pela estrutura molecular dos agentes envolvidos.

Diversas frutas, tais como pomelo (Citrus maxi$m a$ ), laranja-azeda (Citrus aurantium), romã (Punica granatum) e carambola (Averrhoa carambola) contêm agentes inibidores de enzimas de biotransformação, elevando de forma significativa a concentração plasmática de fármacos e favorecendo a potencialização de efeitos, reações adversas ou formação de metabólitos tóxicos (4).

Grande ênfase é dada ao suco de toronja (Citrus paradisi), fruta conhecida como grapefruit, cujos estudos demonstram uma notável capacidade de promover forte inibição do sistema enzimático citocromo P450 3A4 no intestino delgado. 
Quadro 1: Interações fármaco-alimento resultantes de reações químicas

\begin{tabular}{|c|c|c|c|c|}
\hline Fármaco & Classe terapêutica & Alimento & Efeito & Ref \\
\hline Ácido acetilsalicílico & $\begin{array}{l}\text { Anti-inflamatório } \\
\text { não-esteroidal }\end{array}$ & $\begin{array}{l}\text { Dietas à base de frutas, } \\
\text { verduras e fibras }\end{array}$ & $\begin{array}{l}\text { Aumento da excreção devido } \\
\text { à alcalinidade da dieta }\end{array}$ & 8 \\
\hline Amitriptilina & Antidepressivo & $\begin{array}{l}\text { Dietas à base de pães, } \\
\text { biscoitos e carnes }\end{array}$ & $\begin{array}{l}\text { Aumento da excreção devido } \\
\text { à acidez da dieta }\end{array}$ & 8 \\
\hline $\begin{array}{l}\text { Clorpromazina e } \\
\text { flufenazina }\end{array}$ & Antipsicótico & Café, chá mate, caqui e vinhos & $\begin{array}{l}\text { Precipitação na presença } \\
\text { de ácido tânico }\end{array}$ & 8 \\
\hline Morfina & Analgésico opióide & $\begin{array}{l}\text { Dietas à base de pães, } \\
\text { biscoitos e carnes }\end{array}$ & $\begin{array}{l}\text { Aumento da excreção } \\
\text { devido à acidez da dieta }\end{array}$ & 8 \\
\hline Penicilina & Antibiótico & $\begin{array}{l}\text { Dietas à base de frutas, } \\
\text { verduras e fibras }\end{array}$ & $\begin{array}{l}\text { Aumento da excreção devido } \\
\text { à alcalinidade da dieta }\end{array}$ & 8 \\
\hline Prometazina & Anti-histamínico & Café, chá mate, caqui e vinhos & $\begin{array}{l}\text { Precipitação na presença } \\
\text { de ácido tânico }\end{array}$ & 8 \\
\hline Teofilina & Broncodilatador & $\begin{array}{l}\text { Dietas à base de pães, } \\
\text { biscoitos e carnes }\end{array}$ & $\begin{array}{l}\text { Aumento da excreção } \\
\text { devido à acidez da dieta }\end{array}$ & 8 \\
\hline $\begin{array}{l}\text { Tetraciclina e } \\
\text { ceftriaxona }\end{array}$ & Antibiótico & Produtos lácteos & $\begin{array}{l}\text { Complexação do fármaco } \\
\text { com íons divalentes }\end{array}$ & 8,9 \\
\hline
\end{tabular}

Quadro 2: Interações fármaco-alimento com mecanismo de ação específico

\begin{tabular}{|c|c|c|c|c|}
\hline Fármaco & $\begin{array}{l}\text { Classe } \\
\text { terapêutica }\end{array}$ & Alimento & Efeito & Ref \\
\hline $\begin{array}{l}\text { Diazepam, } \\
\text { carbamazepina } \\
\text { e sertralina }\end{array}$ & $\begin{array}{l}\text { Moduladores do } \\
\text { sistema nervoso } \\
\text { central (SNC) }\end{array}$ & Toranja (grapefruit) & $\begin{array}{l}\text { Inibição de enzimas de } \\
\text { biotransformação de fármacos, com } \\
\text { elevação dos seus níveis tóxicos }\end{array}$ & 10 \\
\hline $\begin{array}{l}\text { Inibidores da enzima } \\
\text { monoamina oxidase } \\
\text { (MA0) }\end{array}$ & Antidepressivo & $\begin{array}{l}\text { Queijos maturados, } \\
\text { iogurte, vinhos tintos } \\
\text { e produtos embutidos, } \\
\text { ricos em tiramina }\end{array}$ & $\begin{array}{l}\text { Inibição da enzima que degrada } \\
\text { a tiramina, com elevação de seus níveis } \\
\text { plasmáticos. Efeitos simpaticomiméticos } \\
\text { e crise hipertensiva }\end{array}$ & 11 \\
\hline Isoniazida & Tuberculostático & $\begin{array}{l}\text { Dieta à base de peixes } \\
\text { e frutos do mar, contendo } \\
\text { aminas biogênicas } \\
\text { (histamina, tiramina) }\end{array}$ & $\begin{array}{c}\text { Inibição de enzimas detoxificantes } \\
\text { (MA0 e diamina oxidase). Intoxicação } \\
\text { alimentar e intolerância }\end{array}$ & 12 \\
\hline Mercaptopurina & Antineoplásico & Leite de vaca & Inativação pela enzima xantina oxidase & 13 \\
\hline Varfarina & Anticoagulante & $\begin{array}{l}\text { Alimentos ricos em } \\
\text { vitamina K (ex: brócolis, } \\
\text { couve, couve-de-bruxelas, } \\
\text { salsa e espinafre) }\end{array}$ & $\begin{array}{l}\text { Promoção de coagulação sanguínea e } \\
\text { interferência na efetividade do fármaco }\end{array}$ & 4 \\
\hline Teofilina & Broncodilatador & $\begin{array}{l}\text { Alimentos e bebidas } \\
\text { contendo cafeína (ex: } \\
\text { cafés, chás e chocolates) }\end{array}$ & $\begin{array}{l}\text { Sobrecarga de xantinas, com risco de } \\
\text { toxicidade: distúrbios eletrolíticos e } \\
\text { efeitos no SNC e cardiovascular }\end{array}$ & 4 \\
\hline
\end{tabular}

Em vista dos riscos potenciais, procedimentos determinam que esta combinação seja evitada no uso de muitas classes farmacêuticas, a exemplo de moduladores do sistema nervoso central (diazepam, carbamazepina, sertralina), antagonistas de canais de cálcio (felodipina, verapamil), imunossupressores (ciclosporina) e antirretrovirais (saquinavir), dentre outras (10).
Além de incidir na terapêutica medicamentosa, as interações fármaco-alimento podem dificultar ou diminuir a absorção de nutrientes, afetando o estado nutricional do paciente. Nestes casos, os protocolos clínicos exigem cuidados especiais, como adequação do intervalo entre a administração do medicamento e a ingestão do alimento ou suplementação durante o tratamento (Quadro 3). 
De acordo com Ayo \& Agu (2005), enquanto alguns nutrientes podem interferir na eficácia ou agravar efeitos adversos de fármacos, outros são necessários para aumentar a duração do efeito farmacológico ou mesmo corrigir um efeito colateral do medicamento, por meio de suplementação (3).
Neste contexto, a elucidação de interações fármaco-alimento permite definir critérios para protocolos terapêuticos e o uso correto de medicamentos, sendo que em muitos casos, informações orientativas são contempladas nas bulas dos produtos.

Quadro 3: Interações fármaco-alimento que afetam o estado nutricional e medidas a serem adotadas

\begin{tabular}{|c|c|c|c|c|c|}
\hline fármaco & $\begin{array}{c}\text { classe } \\
\text { terapêutica }\end{array}$ & $\begin{array}{l}\text { nutriente } \\
\text { afetado }\end{array}$ & efeito & $\begin{array}{l}\text { medida dietética } \\
\text { a ser adotada }\end{array}$ & ref \\
\hline $\begin{array}{l}\text { amoxicilina, ampici- } \\
\text { lina, benzilpenicili- } \\
\text { na, cefalotina, cefa- } \\
\text { lexina, ceftriaxona, } \\
\text { ciprofloxacina, clo- } \\
\text { ranfenicol, eritromi- } \\
\text { cina, gentamicina, } \\
\text { oxacilina }\end{array}$ & Antibiótico & Vitaminas K e B12 & $\begin{array}{l}\text { Diminuição da mi- } \\
\text { crobiota intestinal }\end{array}$ & $\begin{array}{l}\text { Produtos lácteos fermentados e } \\
\text { fibras }\end{array}$ & 9 \\
\hline $\begin{array}{l}\text { dexametasona, hi- } \\
\text { drocortisona e pred- } \\
\text { nisona }\end{array}$ & $\begin{array}{l}\text { Anti-inflamatório } \\
\text { esteroidal }\end{array}$ & $\begin{array}{l}\text { Vitaminas A, C, } \\
\text { B1, B6, B9, cálcio, } \\
\text { potássio, fósforo, } \\
\text { magnésio e zinco }\end{array}$ & $\begin{array}{l}\text { Diminuição da ab- } \\
\text { sorção e/ou au- } \\
\text { mento da excreção }\end{array}$ & $\begin{array}{l}\text { Ingestão de frutas nos intervalos } \\
\text { das refeições }\end{array}$ & 14 \\
\hline metotrexato & Antineoplásico & Ácido fólico & $\begin{array}{l}\text { Inibição competi- } \\
\text { tiva da enzima dii- } \\
\text { drofolato-redutase, } \\
\text { com depleção de } \\
\text { folato }\end{array}$ & $\begin{array}{l}\text { Suplemento de ácido fólico e die- } \\
\text { tas ricas em verduras de cor verde } \\
\text { escuro }\end{array}$ & 12 \\
\hline isoniazida & Tuberculostático & Vitamina B6 & $\begin{array}{l}\text { Inibição compe- } \\
\text { titiva da vitamina } \\
\text { B6 pela isoniazida, } \\
\text { com depleção de } \\
\text { vitamina B6 }\end{array}$ & Suplemento de vitamina B6 & 3 \\
\hline óleo mineral & Laxante & $\begin{array}{l}\text { Vitaminas liposso- } \\
\text { lúveis }(A, D, E, K)\end{array}$ & $\begin{array}{l}\text { Diminuição da ab- } \\
\text { sorção e/ou menor } \\
\text { tempo de trânsito } \\
\text { intestinal }\end{array}$ & $\begin{array}{l}\text { Intervalos adequados entre a ad- } \\
\text { ministração do medicamento e as } \\
\text { refeições }\end{array}$ & 9,15 \\
\hline $\begin{array}{l}\text { omeprazol, ranitidi- } \\
\text { na e cimetidina }\end{array}$ & Anti-ulceroso & Vitamina B12 & $\begin{array}{l}\text { Diminuição da ab- } \\
\text { sorção }\end{array}$ & $\begin{array}{l}\text { Suplemento de vitamina B12 e } \\
\text { dieta rica em alimentos de ori- } \\
\text { gem animal }\end{array}$ & 14 \\
\hline
\end{tabular}

\section{CONCLUSÃO}

A interação entre fármacos e alimentos é um tema cada vez mais relevante à equipe multidisciplinar de saúde, já que os alimentos são substâncias químicas diversificadas e constantemente disponíveis no organismo. Principalmente no que se refere às adversidades destas interações, é muito importante que os profissionais participem de programas educacionais e implementem procedimentos de prevenção e resolução de problemas.

Uma maior atenção deve ser dada aos casos de tratamentos crônicos e dietas monótonas, pois a biodisponibilidade do fármaco, bem como a de nutrientes, é essencial para o processo de cura de doenças. 


\section{REFERÊNCIAS}

1. Gauthier I, Malone M. Drug-food interactions in hospitalised patients. Methods of prevention. Drug Safety, 1998. 18(6):383-93.

2. Machado T, Machado J. Interação alimentos e medicamentos: um assunto despercebido pela população. Infarma, 2008. 20(9/10):46-8.

3. Ayo JA, Agu H. Food and drug interactions: its side effects. Nutr Food Sci, 2005. 35(4):243-252.

4. Bushra R, Aslam N, Khan AY. Food-drug interactions. Oman Med J, 2011. 26(2):77-83.

5. Genser D. Food and drug interaction: consequences for the nutrition/health status. Ann Nutr Metab, 2008. 52(1):29-32.

6. Schmidt LE, Dalhoff K. Food-drug interactions. Drugs, 2002. 62(10):1481-1502.

7. Heird WC. Necessidades Nutricionais. In: Behrman RE, Kliegman RM, Jeson HB. Nelson: Tratado de Pediatria. Rio de Janeiro: Elsevier. 2005. p.167-71.

8. Schweigert ID, Pletsch MU, Dallepianne LB. Interação medicamento-nutriente na prática clínica. Rev Bras Nutr Clin, 2008. 23(1):72-7.
9. Lopes EM, Oliveira EAR, Lima LHO, Formiga LMF, Freitas RM. Interações fármaco-alimento/nutriente potenciais em pacientes pediátricos hospitalizados. Rev Ciên Farm Básica Apl, 2013. 34(1):131-35.

10. Dahan A, Altman H. Food-drug interaction: grapefruit juice augments drug bioavailability - mechanism, extent and relevance. Eur J Clin Nutr, 2004. 58:1-9.

11. Volz HP, Gleiter CH. Monoamine oxidase inhibitors. A perspective on their use in the elderly. Drugs Aging, 1998. 13(5):341-55.

12. Masuko K, Tohma S, Matsui T. Potential food-drug interactions in patients with rheumatoid Arthritis. Int J Rheum Dis, 2013. 16:122-28.

13. Lemos ML, Hamata L, Jennings S, Leduc T. Interaction between mercaptopurine and milk. J Oncol Pharm Practice, 2007. 13(4):237-40.

14. Gomez R, Venturini CD. Interação entre alimentos e medicamentos. $1^{\mathrm{a}}$. ed. Porto Alegre: Letra e Vida, 2009.

15. Moura MRL, Reyes FGR. Interação fármaco-nutriente: uma revisão. Rev Nutr, 2002. 15(2):223-238. 